\title{
Fuzzy Weighted Associative Classifier: A PREDICTIVE TECHNIQUE FOR HEALTH CARE DATA MINING
}

\author{
Sunita Soni ${ }^{1}$ and O.P.Vyas ${ }^{2}$ \\ ${ }^{1}$ Associate Professor,Bhilai Institute of Technology, Durg-491 001, Chhattisgarh, India \\ Sunitasoni74@gmail.com \\ ${ }^{2}$ Professor, Indian Institute of Information Technology, Allahabad, Uttar Pradesh, India \\ dropvyasegmail.com
}

\begin{abstract}
In this paper we extend the problem of classification using Fuzzy Association Rule Mining and propose the concept of Fuzzy Weighted Associative Classifier (FWAC). Classification based on Association rules is considered to be effective and advantageous in many cases. Associative classifiers are especially fit to applications where the model may assist the domain experts in their decisions. Weighted Associative Classifiers that takes advantage of weighted Association Rule Mining is already being proposed. However, there is a so-called "sharp boundary" problem in association rules mining with quantitative attribute domains. This paper proposes a new Fuzzy Weighted Associative Classifier (FWAC) that generates classification rules using Fuzzy Weighted Support and Confidence framework. The naïve approach can be used to generating strong rules instead of weak irrelevant rules. where fuzzy logic is used in partitioning the domains. The problem of Invalidation of Downward Closure property is solved and the concept of Fuzzy Weighted Support and Fuzzy Weighted Confidence frame work for Boolean and quantitative item with weighted setting is generalized. We propose a theoretical model to introduce new associative classifier that takes advantage of Fuzzy Weighted Association rule mining.
\end{abstract}

\section{Keywords}

Associative Classifiers, Fuzzy Weighted Association Rule, FWAC, Fuzzy weighted support, Fuzzy weighted Confidence.

\section{INTRODUCTION}

Associative Classification is an integrated framework of Association Rule Mining (ARM) and Classification. A special subset of association rules whose right-hand-side is restricted to the classification class attribute is used for classification.

The traditional ARM was designed considering that items have same importance and in the database simply their presence or absence is mentioned. In several problem domains it does not make sense to assign equal importance to all the items particularly in predictive modeling system

DOI : $10.5121 /$ ijcseit.2012.2102 
where attributes have different prediction capability. For example in medical domain predicting the probability of heart disease, the attribute prior-stroke is having more impact than the attribute BMI (Body Mass Index). The concept of weighted association rule mining is used to deal with the case where items are assigned a weight to reflect their importance. To deal with the situation the authors have proposed a new Weighted Associative Classifier (WAC) that generates classification rules using weighted support and Confidence framework [1].

Another problem in the medical database as well as databases from other applications is that most of the attributes are associated with quantitative domains such as BMI, Age, BloodPressure, etc., which are very common in many real applications, association rule mining usually needs to partition the domains in order to apply the Apriori-type method. Thus, a discovered rule $X \rightarrow Y$ reflects association between interval values of data items. Examples of such rules are $\{($ Age,">62”), (BMI,“45”), (Blood_pressure,"95-135") $\} \rightarrow$ (Heart_Disease) and (Income[20,000$30,000] \rightarrow$ Age[20-30]) and so on. Apart from domain discretization techniques for association rule mining, fuzzy logic is considered as suitable solution to deal with the "sharp boundary" problem. This gives rise to the notion of Fuzzy Association Rules (FAR). These rules are richer and of certain natural language nature. For example, (old Age, high Obesity and high Blood_Pressure) $\rightarrow$ (chance of Heart Disease) and (medium, Income) $\rightarrow$ (young Age) are fuzzy association rules, where X's and Y's are fuzzy sets with linguistic terms (i.e., old, high, hyper medium, and young). Building an associative classifier based upon fuzzy association rules provides two advantages: one is the need to mine large datasets with quantitative domains; the other is to generate classification rules with more general semantics and linguistic expressiveness [3].

This paper proposes a new Fuzzy Weighted Associative Classifier (FWAC) that generates classification rules using Fuzzy Weighted Support and Confidence framework. The naïve approach can be used to generate strong rules instead of weak irrelevant rules. We discussed the importance of Fuzzy Weighted Association rule in classification problem.

In section 2, we have discussed the concept of association rule mining and fuzzy weighted associative classifiers. In section 3 we described some new formulae and given new definitions for the same. In section 4, downward closure properties for Fuzzy weighted version of association rule mining is being discussed. Section 5 discuss some of the application area that can be benefited with the proposed concept. In section 6, conclusion and future work of this paper is given.

\section{Related Work}

\subsection{Association Rule Mining}

Let $I=\left\{i_{1}, i_{2} \ldots i_{\mathrm{n}}\right\}$ be a set of $n$ distinct literals called items. $D$ is a set of variable length transactions over $I$. Each transaction contains a set of items $i_{1}, i_{2} \ldots i_{\mathrm{k}} \in I$. A transaction has an associated unique identifier called TID. An association rule is an implication of the form $A \Rightarrow B$ (or written as $A \rightarrow B$ ), where $A, B \subseteq I$, and $A \cap B=\varnothing$. $A$ is called the antecedent of the rule and $B$ is called the consequent of the rule. The rule $X \Rightarrow Y$ has a support $s$ in the transaction set $D$ if $s \%$ of the transactions in $D$ contain $X \cup Y$. In other words, the support of the rule is the probability that $X$ and $Y$ hold together among all the possible presented cases. It is said that the rule $X \Rightarrow Y$ holds 
in the transaction set $D$ with confidence $c$ if $c \%$ of transactions in $D$ that contain $X$ also contain $Y$. In other words, the confidence of the rule is the conditional probability that the consequent $Y$ is true under the condition of the antecedent $X$. The problem of discovering all association rules from a set of transactions $D$ consists of generating the rules that have a support and confidence greater than given thresholds. These rules are called strong rules, and the framework is known as the support confidence framework for association rule mining.

\subsubsection{Weighted Association Rule Mining}

A weighted association rule (WAR) is an implication $\mathrm{X} \rightarrow \mathrm{Y}$ where $\mathrm{X}$ and $\mathrm{Y}$ are two weighted items. A pair $\left(i_{j}, w_{j}\right)$ is called a weighted item where $i_{j} \in I$ and $w_{j} \in W$ is the weight associated with the item $i_{j}$. A transaction is a set of weighted items where $0<w_{j}<=1$. Weight is used to show the importance of the item. For example in the context of stock market prices, some stocks have much higher value than others and might appreciate a lot more than other stocks in a comparable time period. In the supermarket context, some items like jewellery, designer clothes, etc. are of much higher value than trivia like bubblegum or candy. Rules involving jewellery may have less support than those involving candy but are much more significant in terms of the revenue (and consequently profit) earned by the store.

In weighted association rule mining problem each item is allowed to have a weight. The goal is to steer the mining process to those significant relationships involving items with significant weights rather than being flooded in the combinatorial explosion of insignificant relationships [8].

\subsubsection{Fuzzy Association Rule Mining (FARM).}

A data mining for Discovering Fuzzy Association Rules is proposed in [11]. The authors have given the technique to find Fuzzy Association Rules without using the user supplied support values which are often hard to determine. The other unique feature of the work is that the conclusion of a fuzzy association rule can contain linguistic terms. The experimental result shows that the algorithm is capable of discovering both positive and negative fuzzy association rules in an effective manner from real life database.

In [5] the authors have proposed a model to find the fuzzy association rules in fuzzy transaction database. The model is found to be useful technique to find the patterns in data in the presence of imprecision, either because data are fuzzy in nature or because we must improve their semantics. Authors have also discussed some of the applications of the scheme, paying special attention to the discovery of fuzzy association rules in relational database.

\subsubsection{Fuzzy Weighted Association Rule Mining}

Fuzzy Weighted Association Rule Mining with Weighted Support and Confidence Framework is proposed in [2]. The authors have addressed the issue of invalidation of downward closure property (DCP) in weighted association rule mining where each item is assigned a weight according to their significance. Formulae for fuzzy weighted support and fuzzy weighted confidence for Boolean and quantitative items with weighted settings is proposed. The methodology follows an Apriori like approach and avoids the pre and post processing as opposed to most weighted ARM algorithm, thus eliminating the extra steps during rules generation. 
A new algorithm which is applicable to Normalized and unnormalized case is proposed in [11] In this paper the authors have introduced the problem of mining Weighted Quantitative Association rules based on Fuzzy approach. Using the fuzzy set concept, the discovered rules are more understandable to a human. Two different definition of weighted Support with and without normalization is proposed.

\subsubsection{Incorporating Weight in ARM}

The concepts of assigning weight to the attribute have never been utilized in medical domain. In Super marker context the concept of has been used to assigned more weight to the item that gives more profit per unit sale. Other example in the context of stock market prices, some stocks have much higher value than others and might appreciate a lot more than other stocks in a comparable time period.

In traditional association rule mining (ARM) model only item's presence or absence in the transaction is mentioned and its significance is not considered at profit point of view. To deal with the weighted setting environment, an algorithm called WARM (Weighted Association Rule Mining) is proposed [8] in which the item's weighted support is measured instead of calculating only support. The weighted support is a measure of significance of an item at cost point of view. The goal of using weighted support is to make use of the weight in the mining process and prioritize the selection of target itemsets according to their significance in the dataset, rather than their frequency alone. An itemset is denoted large if its support is above a predefined minimum support threshold. In the WARM context, an itemset is said to be significant if its weighted support is above a pre-defined minimum weighted support threshold. The threshold values specified by the user are significance of item at cost point of view.

\subsubsection{Utilizing Weight in Medical Domain}

Motivation to utilized weight in medical domain is from supermarket scenario where a weight is assigned to each of the items according to the profit it generates to the store, rather than simply counting and calculating the percentage of transactions that contain items. In medical domain also some of the symptoms have much impact to predict particular disease. For example in predicting the probability of heart disease, the attribute prior-stroke is having more impact than the attribute BMI (Body Mass Index).The experience of expert doctor can be utilized to assign weight to the different symptoms in medical domain. This is also the way to utilize the experience of Domain Expert in Prediction Model. In medical domain the algorithm WARM can be utilized to generate weighted CAR rule. So in this paper we have used the WARM to incorporate attribute importance instead of considering the weight separately in the algorithm.

\subsubsection{Fuzziness of Quantitative Attribute}

In ARM model when the data are quantitative such as income, age, price, etc., which are very common in many real applications, association rule mining needs to descitization of domain to convert it in to nominal domain. And ultimately apply the Apriori-type method. Thus, association rule like $X \rightarrow Y$ reflects association between nominal values of data items. Examples of such rules are "(Age, old), (BP, high) $\rightarrow$ (Heart_Disease, yes) , "(Income, Low) $\rightarrow$ (Age, medium)", and so on. Such the mining results are affected by how the intervals are partitioned, particularly for data values around interval boundaries. That is the so-called "sharp boundary" problem. 
Subsequently, the result of associative classification may also be affected in terms of accuracy and understandability.

In medical domain there are number of quantitative attributes suffers from crisp boundary problem. For example attributes for example if in a particular record the BMI (Body mass Index) is 41 them according to following discritization rule

$$
\begin{aligned}
& \text { BMI[26-30] } \rightarrow \text { Obesity="mild" } \\
& \text { BMI[31-40] } \rightarrow \text { Obesity="moderate" } \\
& \text { BMI[40-*] } \rightarrow \text { Obesity="sever" }
\end{aligned}
$$

The patient is considered to be severely obese. This may not give a correct result because of sharp boundary problem. Instead by applying fuzzy logic the patient is partially belonging to each fuzzy set. Hence the patient membership value to the fuzzy set should be $(\mu$ (obesity, "mild") $=0.1, \mu$ (obesity, "moderate") $=0.3, \mu$ (obesity, "sever") $=0.6$ ). To deal with crisp boundary problem of quantitative attribute in ARM model the [2] proposed the Fuzzy WARM (FWARM) Algorithm and redefine the weighted support and weighted confidence to adapt in Fuzzy environment. In Fuzzy Weighted Association Rule Mining (FWARM) model the Fuzzy Weighted Support (FWS) and Fuzzy Weighted Confidence framework is proposed to mine Fuzzy Weighted Association Rule. The algorithm FWARM can be used to generate the CAR rules in Fuzzy Weighted environment.

\section{Problem Definition}

In this paper we have proposed two important modifications (weight of an attribute and fuzzyfication of quantitative attributes) in Associative Classifier to improve Prediction Accuracy in Medical Domain. Hence the problem definition consists of the terms and basic concepts to define Fuzzy Attribute Weight (FAW), Fuzzy Attribute set Transaction Weight (FASTW), Fuzzy Attribute Set Weight (FASW) Fuzzy Weighted Support (FWS) and Fuzzy Weighted Confidence (FWC) for Fuzzy Weighted Associative Classifiers (FWAC). Technique for Fuzzy Weighted Association Rule Mining is known as (FWARM).

\subsection{Associative Classifiers.}

Given a set of cases with class labels as a training set, classification is to build a model (called classifier) to predict future data objects for which the class label is unknown.

Associative Classification is an integrated framework of Association Rule Mining (ARM) and Classification. A special subset of association rules whose right-hand-side is restricted to the classification class attribute is used for classification. This subset of rules is referred as the Class Association Rules (CARs). Recent studies propose the extraction of a set of high quality association rules from the training data set, which satisfy certain user-specified frequency and confidence thresholds. Effective and efficient classifiers have been built by careful selection of rules, e.g., CBA, CAEP and ADT. Such a method takes the most effective rule(s) from among all the rules mined for classification. Since association rules explore highly confident associations among multiple variables, it may overcome some constraints introduced by a decision-tree induction method, which examines one variable at a time. Extensive performance studies [11] show that association based classification may have better accuracy in general [9]. 
International Journal of Computer Science, Engineering and Information Technology (IJCSEIT), Vol.2, No.1, February 2012

\subsection{Fuzzy Weighted Associative Classifiers.}

A fuzzy dataset consists of fuzzy relational database $D=\left\{r_{1}, r_{2}, r_{3} \ldots . r_{i} \ldots r_{n}\right\}$ with a set of attributes $\mathrm{I}=\left(\mathrm{I}_{1}, \mathrm{I}_{2}, \ldots \ldots \mathrm{I}_{\mathrm{m}}\right\}$, each $\mathrm{I}_{\mathrm{K}}$ can be associated with a set of linguistic labels $\mathrm{L}=\left\{1_{1}, 1_{2}\right.$, $\left.\ldots \ldots \mathrm{l}_{\mathrm{L}}\right\}$ for example $\mathrm{L}=\{$ young, Middle, Old $\}$.Let each $\mathrm{I}_{\mathrm{k}}$ is associated with fuzzy set $\mathrm{F}_{\mathrm{k}}=\left\{\left(\mathrm{I}_{\mathrm{k}}, \mathrm{l}_{1}\right)\right.$, $\left.\left(\mathrm{I}_{\mathrm{k}}, \mathrm{l}_{2}\right), \quad\left(\mathrm{I}_{\mathrm{k}}, \mathrm{l}_{3}\right), \ldots \ldots\left(\mathrm{I}_{\mathrm{k}}, \mathrm{l}_{\mathrm{L}}\right)\right\}$. So that a new Fuzzy Database $\mathrm{D}$ ' is defined as $\left\{\left(\mathrm{I}_{1}, \mathrm{l}_{1}\right) \ldots\left(\mathrm{I}_{1}, \mathrm{l}_{\mathrm{L}}\right) \ldots\left(\mathrm{I}_{\mathrm{k}}\right.\right.$, $\left.1_{1)}, \ldots\left(I_{k}, l_{L}\right) \ldots\left(I_{m}, l_{1}\right) \ldots\left(I_{m}, l_{L}\right)\right\}$.Each attribute $I_{i}$ in a given transaction $t_{k}$ is associated (to some degree) with Several fuzzy sets. The degree of association is given by a membership degree in the range $[0 . .1] . t_{k}\left[\mu\left(I_{i}, l_{j}\right)\right]$ will denote the degree of membership for Fuzzy Attribute $I_{i}$ to fuzzy set $\mathrm{l}_{\mathrm{j}}$ in transaction $\mathrm{t}_{\mathrm{k}}$.

Table-1 Data Base with continuous domain

\begin{tabular}{|c|c|c|c|c|}
\hline (D) R_ID & Age & $\begin{array}{l}\text { Blood } \\
\text { Pressure } \\
\text { (BP) }\end{array}$ & $\begin{array}{l}\text { BMI } \\
\text { (Obesity) }\end{array}$ & Heart_Disease(H_D) \\
\hline 1 & 42 & $90-130$ & 40 & Yes \\
\hline 2 & 62 & $80-120$ & 28 & No \\
\hline 3 & 55 & $82-122$ & 40 & Yes \\
\hline 4 & 62 & $92-135$ & 50 & Yes \\
\hline 5 & 45 & $95-135$ & 30 & No \\
\hline
\end{tabular}

Table-2 Transformed Binary Database D' from D

\begin{tabular}{|c|c|c|c|c|c|c|c|c|c|c|}
\hline \multirow{2}{*}{$\begin{array}{l}\text { (D') } \\
\text { R_ID }\end{array}$} & \multicolumn{3}{|c|}{ Age } & \multicolumn{3}{|c|}{ Blood Pressure(BP) } & \multicolumn{3}{|c|}{ BMI(Obesity) } & \multirow{2}{*}{$\begin{array}{l}\text { Heart } \\
\text { Disease } \\
\text { ( H_D) }\end{array}$} \\
\hline & $\stackrel{\infty}{\Xi}$ & $\frac{\ddot{v}}{\stackrel{0}{*}}$ & 궁 & 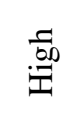 & 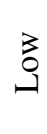 & 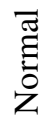 & $\frac{7}{3}$ & $\begin{array}{l}\frac{\mathbb{\pi}}{\tilde{J}} \\
\frac{0}{\Delta} \\
\Sigma\end{array}$ & $\begin{array}{l}\stackrel{D}{0} \\
\vec{D} \\
\omega\end{array}$ & \\
\hline 1 & 0 & 1 & 0 & 1 & 0 & 0 & 0 & 1 & 0 & $\mathrm{Y}$ \\
\hline 2 & 0 & 0 & 1 & 0 & 0 & 1 & 0 & 1 & 0 & $\mathrm{~N}$ \\
\hline 3 & 0 & 1 & 0 & 1 & 0 & 0 & 0 & 1 & 0 & $\mathrm{Y}$ \\
\hline 4 & 0 & 0 & 1 & 1 & 0 & 0 & 0 & 0 & 1 & $\mathrm{Y}$ \\
\hline 5 & 0 & 1 & 0 & 1 & 0 & 0 & 1 & 0 & 0 & $\mathrm{~N}$ \\
\hline
\end{tabular}




\begin{tabular}{|c|c|c|c|c|c|c|c|c|c|c|}
\hline \multirow[t]{2}{*}{$\mathrm{D}^{\prime \prime}$} & \multicolumn{3}{|c|}{ Age } & \multicolumn{3}{|c|}{ BP } & \multicolumn{3}{|c|}{ BMI } & \multirow[t]{2}{*}{ H_D } \\
\hline & $\begin{array}{l}\stackrel{0}{0} \\
\Xi \\
0 \\
\stackrel{\lambda}{2}\end{array}$ & $\frac{\ddot{\theta}}{\frac{0}{g}}$ & 긍 & 韋 & 苂 & $\begin{array}{l}\overline{\widetilde{J}} \\
\text { Z } \\
\text { Z }\end{array}$ & $\bar{z}$ & 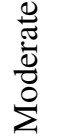 & $\begin{array}{l}\stackrel{0}{0} \\
\vec{\Delta} \\
\tilde{D}\end{array}$ & \\
\hline 1 & 0.2 & 0.7 & 0.1 & 0.4 & 0 & 0.6 & 0.6 & 0.3 & 0.1 & $\mathrm{Y}$ \\
\hline 2 & 0.0 & 0.3 & 0.7 & 0.1 & 0.1 & 0.8 & 0.8 & 0.1 & 0.1 & $\mathrm{~N}$ \\
\hline 3 & 0.1 & 0.3 & 0.6 & 0.2 & 0.0 & 0.8 & 0.6 & 0.3 & 0.1 & $\mathrm{Y}$ \\
\hline 4 & 0.0 & 0.3 & 0.7 & 0.5 & 0.0 & 0.5 & 0.1 & 0.2 & 0.7 & $\mathrm{Y}$ \\
\hline 5 & 0.1 & 0.8 & 0.1 & 0.6 & 0.0 & 0.4 & 0.7 & 0.2 & 0.1 & $\mathrm{~N}$ \\
\hline
\end{tabular}

Table-3 Database D”, with Fuzzy Items.

Table 1 shows the Example database D with continuous Domain of quantitative attribute. In Table 2 the transformed binary database (D') is shown in which the quantitative attributes have been partitioned by converting it into categorical attribute. Consider attribute Age in Table 1 again, three new attributes (e.g. ((Age, young), (Age, middle) and (Age, old) in place of Age may be used to constitute a new database $\left(\mathrm{D}^{\prime \prime}\right)$ with partial belongings of original attribute values to each of the new attributes. Table 3 illustrates an example of the new database obtained from the original database, given fuzzy sets $\{$ Young, Middle, Old $\}$ as characterized by membership functions shown in Figure 1 for attribute age. Similarly the other quantitative attribute ie Blood pressure and BMI (Obesity) are also partitioned and membership values are assigned by using corresponding membership function.

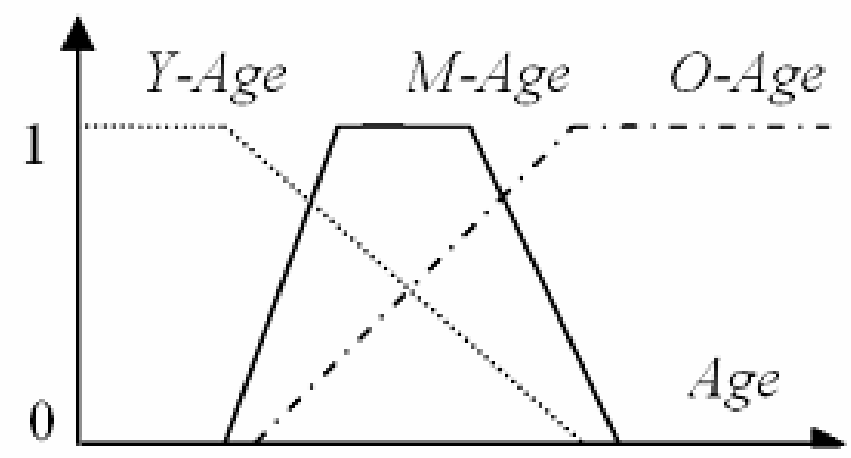

Figure 1: Fuzzy Sets $Y$-Age, $M$-Age and $O$-Age.

Here Fuzzy logic is incorporated to split the domain of quantitative attribute into intervals, and to define a set of meaningful linguistic labels represented by fuzzy sets and use them as a new domain. In this case it is possible that one item may appear with different label of same attribute. Hence the item sets are needs to be restricted to contain at most one item set per attribute because 
otherwise the rules of the form $\{($ Age, Middle),(Age, old)..... $\Rightarrow$ class_label $\}$ have no meaning. The triangle and trapezoidal are the two important membership functions that can be used to find the degree of association for the different attribute.

Definition 1. Fuzzy Attribute Weight: We assign a weight $W\left(I_{i}, l_{j}\right)$ to each fuzzy Item $I\left(I_{i}, l_{j}\right)$ where $(1 \leq \mathrm{i} \leq \mathrm{n}),(1 \leq \mathrm{j} \leq \mathrm{L})$ and $(0 \leq \mathrm{w} \leq 1)$. Table 4 shows the random weight assigns to different fuzzy attribute for heart disease

\begin{tabular}{|l|l|l|}
\hline S. No. & Symptoms & Weights \\
\hline 1 & (Age, young) & 0.1 \\
2 & (Age, middle) & 0.2 \\
3 & (Age, old) & 0.6 \\
4 & (BP, Norma) & 0.3 \\
5 & (BP, Low) & 0.2 \\
6 & (Bp, High) & 0.7 \\
7 & (BMI, Mild) & 0.3 \\
8 & (BMI, Moderate) & 0.5 \\
9 & (BMI, severe) & 0.7 \\
\hline
\end{tabular}

Table-4 Weight of symptoms for heart disease (attribute weight).

Definition 2. Fuzzy Attribute set Transaction Weight: Weight of attribute set $X$ a particular transaction $t_{k}$ is denoted by $t_{k}[F A T W(X)]$ and is calculated as the product of membership degree of attribute in given fuzzy set in the transaction $t_{k}$ and weight of fuzzy attribute; of all enclosing Fuzzy attribute in the set. And is given by

$$
\left.\operatorname{tk}[\operatorname{FASTW}(\mathrm{X})]=\quad \prod\left(\forall\left(\mathrm{I}_{\mathrm{i}}, \mathrm{l}_{\mathrm{j}}\right) \in \mathrm{X}\right) \underset{\mathrm{i}=1}{[\mathrm{X} \mid} \underset{\mathrm{t}}{\mathrm{t}}\left[\mu\left(\mathrm{I}_{\mathrm{i}}, 1_{\mathrm{j}}\right)\right] \times \mathrm{W}\left(\mathrm{I}_{\mathrm{i}}, 1_{\mathrm{j}}\right)\right]
$$

Example 2: Consider the 2 attribute set (Age , old), (BP, high ) in transaction1

FASTW $(($ Age, old $),(B P$, high $))=(0.1 \times 0.6)(0.4 \times 0.7)=0.34$

Definition 3. Fuzzy Attribute Set Weight: Fuzzy Weight of attribute set $X$ is calculated as sum of FASTW all transaction and is denoted by FASW(X). And is given by

$$
\operatorname{FASW}(\mathrm{X})=\quad \sum_{\mathrm{k}=1}^{\left|\mathrm{D}^{\prime \prime}\right|} \mathrm{t}_{\mathrm{k}}[\operatorname{FASTW}(\mathrm{X})]
$$


$\operatorname{FSAW}(\mathrm{X})=\sum_{\mathrm{k}=1}^{\left|\mathrm{D}^{\prime \prime}\right|} \prod_{\mathrm{i}=1}^{|\mathrm{X}|}\left(\forall\left(\mathrm{I}_{\mathrm{i}}, \mathrm{l}_{\mathrm{j}}\right) \in \mathrm{X}\right) \quad\left[\operatorname{tk}\left[\mu\left(\mathrm{I}_{\mathrm{i}}, \mathrm{l}_{\mathrm{j}}\right)\right] \times \mathrm{W}\left(\mathrm{I}_{\mathrm{i}}, \mathrm{l}_{\mathrm{j}}\right)\right]$

Example 3: Consider the 2 attribute set (Age, old), (BP, high ) .

FASW $(($ Age, old $),(B P$, high $))=[(0.1 \times 0.6)(0.4 \times 0.7)+(0.7 \times 0.6)(0.1 \times 0.7)+(0.6 \times 0.6)(0.2 \times 0.7)$ $+(0.7 \times 0.6)(0.5 \times 0.7)+(0.1 \times 0.6)(0.6 \times 0.7)]=2.34$

Definition 4. Fuzzy Weighted Support: In associative classification rule mining, the association rules are not of the form $\mathrm{X} \rightarrow \mathrm{Y}$ rather they are subset of these rules where $\mathrm{Y}$ is the class label.

Fuzzy Weighted support FWS of rule $\mathrm{X} \rightarrow$ Class_label, where $\mathrm{X}$ is set of non empty subsets of fuzzy weighted attribute. Fuzzy Weighted Support FWS of a rule $\mathrm{X} \rightarrow$ Class_label is calculated as sum of weight of all transaction in which the given class label is true, divided by total number of transaction, denoted by FWS $(\mathrm{X} \rightarrow$ Class_label $)$. And is given by

$$
\begin{aligned}
& \sum \forall \mathrm{t}_{\mathrm{k}} \text { having } \mathrm{t}_{\mathrm{k}}[\mathrm{FASTW}(\mathrm{X})] \\
& \text { Given } \\
& \text { class_label }
\end{aligned}
$$

$\mathrm{FWS}(\mathrm{X} \rightarrow$ Class_label $)=$

Number of records in D",

where $t_{\mathrm{k}}$ is all transaction for which the given class_label is true

$$
\begin{aligned}
& |\mathrm{X}| \\
& \sum \forall \mathrm{t}_{\mathrm{k}} \text { having } \quad \prod \forall\left(\mathrm{I}_{\mathrm{i}}, \mathrm{l}_{\mathrm{j}}\right) \in \mathrm{X}\left[\mu\left(\mathrm{I}_{\mathrm{i}}, 1_{\mathrm{j}}\right) \times \mathrm{W}\left(\mathrm{I}_{\mathrm{i}}, 1_{\mathrm{j}}\right)\right] \\
& \text { Given } \quad \mathrm{i}=1 \\
& \mathrm{FWS}(\mathrm{X} \rightarrow \text { Class_label })= \\
& \text { class_label }
\end{aligned}
$$

Example 4: Consider the attribute set $\mathrm{X}=[($ Age, old $),(\mathrm{BP}$, high $)]$ and a rule $\mathrm{r}=[($ Age, old $),(\mathrm{BP}$, high $\rightarrow$ (Heart_disease= "yes") the Fuzzy Weighted Support of a rule is given by FWS $(($ Age, old $),(B P$, high $) \rightarrow$ (Heart_disease="yes"))

$[(0.1 \times 0.6)(0.4 \times 0.7)+(0.6 \times 0.6)(0.2 \times 0.7)+(0.7 \times 0.6)(0.5 \times 0.7)]$

\section{5}

$\mathrm{FWS}(\mathrm{r})=0.27(27 \%)$

Definition 5. Fuzzy Weighted Confidence: Fuzzy Weighted Confidence of a rule $X \rightarrow Y$ where $\mathrm{Y}$ represents the Class label can be defined as the ratio of Fuzzy Weighted Support of $(\mathrm{X} \cup \mathrm{Y})$ and Fuzzy Weighted Support of (X). And is given by

$$
\text { Fuzzy Weighted Support }(X \cup Y)
$$

Fuzzy Weighted

Confidence $=$

$$
\text { Fuzzy Weighted Support (X) }
$$




$$
\begin{aligned}
& \text { |X| }
\end{aligned}
$$

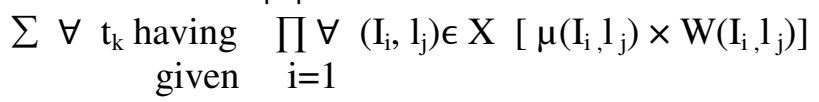

$\operatorname{FWC}(\mathrm{X})=$

$$
\sum_{\mathrm{K}=1}^{\left|\mathrm{D}^{\prime \prime}\right|} \prod_{\mathrm{i}=1}^{|\mathrm{X}|}\left(\forall\left(\mathrm{I}_{\mathrm{i}}, \mathrm{l}^{\mathrm{j}}\right) \in \mathrm{X}\right)\left[\mu\left(\mathrm{I}_{\mathrm{i}}, 1_{\mathrm{j}}\right) \times \mathrm{W}\left(\mathrm{I}_{\mathrm{i}}, \mathrm{l}_{\mathrm{j}}\right)\right]
$$

Example 5: Consider the attribute set $X=[($ Age, old $),(B P$, high $)]$ and a rule $r=[($ Age, old $),(B P$, high $) \rightarrow($ Heart_disease= "yes") the Fuzzy Weighted Confidence of a rule is given by

FWC $[($ Age, old $),($ BP, high $) \rightarrow$ Heart_disease="yes") $]=$ $[(0.1 \times 0.6)(0.4 \times 0.7)+(0.6 \times 0.6)(0.2 \times 0.7)+(0.7 \times 0.6)(0.5 \times 0.7)]$

$[(0.1 \times 0.6)(0.4 \times 0.7)+(0.7 \times 0.6)(0.1 \times 0.7)+(0.6 \times 0.6)(0.2 \times 0.7)+(0.7 \times 0.6)(0.5 \times 0.7)+(0.1 \times 0.6)(0.6 \times 0$. 7)]

$$
\mathrm{FWC}(\mathrm{r})=1.37 / 2.34
$$

$\mathrm{FWC}(\mathrm{r})=0.585(58 \%)$

\section{WEIGHTED DOWNWARD CLOSURE PROPERTY}

In a classical Apriori algorithm it is assumed that if the itemset is large, then all its subsets should also be large and is called Downward Closure Property (DCP). This helps algorithm to generate large itemsets of increasing size by adding items to itemsets that are already large. In the weighted ARM case where each item is assigned weight, the DCP does not hold. To solve the problem of invalidation of DCP, the new framework, "Fuzzy weighted support framework" is designed in [2]. The authors have proved that using Fuzzy weighted support the "weighted downward closure property" retains. the authors have prove that if an itemset $\{A C\}$ is not significant then its superset say $\{A C E\}$ is impossible to be significant hence no need to calculate its Fuzzy weighted support. To generate the frequent item set in the proposed method, the Apriori algorithm has been used and instead of using "support - large" framework the new framework of "Fuzzy weighted support Framework" has been used.

\section{ApPlications}

- Medical Application: In medical database most of the attributes are quantitative in nature. Descritization of these attributes will suffer crisp boundary problem Hence fuzzy environment can be used. Assigning weights to the symptoms to improves prediction accuracy compare to the traditional Associative classifiers.

- Business Application: Fuzzy weighted environment is suitable for customer classification based upon their purchasing habit. In fuzzy transactional Database [2] the weighted concept can be used to assign External utility to the item in the supermarket.

- Web Mining: In web mining visitor page dwelling time can be used to assign weight. 
International Journal of Computer Science, Engineering and Information Technology (IJCSEIT), Vol.2, No.1, February 2012

- Classification Problem: Utility of the Fuzzy weighted Associative Classifiers is not limited to health care rather it can be applied in any domain to improve the prediction accuracy.

\section{CONCluSion \& Future Work}

This work presents a new foundational approach to Fuzzy Weighted Associative Classifiers where quantitative attributes are discritized to get transformed binary database. In such data base each record fully belongs to only one fuzzy set. Such database will suffer the crisp boundary problem. To deal with crisp boundary problem of quantitative attribute in ARM model the Fuzzy WARM (FWARM) Algorithm has been proposed and redefine the weighted support and weighted confidence to adapt in Fuzzy environment.

Each Fuzzy attribute is allowed to have weight depending upon their importance in predicting the class labels. A Conceptual model has been presented that allows development of an efficient and applicable algorithm in future that can capture real-life situations and can produce more accurate classifiers such as in Medical data mining. It has already been proved that, by assigning weights to Fuzzy items and using FWARM, the selection of significant item sets is steered to those item sets containing or having relationships to high weight items. Hence using Fuzzy weighted Association Rule as a Classification rule will improve the classification accuracy. In future work the proposed concept needs to be implemented to find out how much accuracy is improved by adapting the above concept. One of existing associative classifiers is to be chosen or new algorithm needs to be developed that can be integrated with Fuzzy weighted association rule miner.

\section{REFERENCES}

[1] S. soni, O.P. Vyas, J. pillai, Associative Classifier Using Weighted Association Rule, Symposium 2009 World Congress on Nature \& Biologically Inspired Computing (NaBIC 2009) page(s):14921496.

[2] M. Suleman Khan, Maybin Muyeba, M.Frans Coenen, Fuzzy weighted Association Rule Minging with weighted Support and Confidence framework. 2009

[3] Zuoliang Chen, Guoqing Chen, BUILDING AN ASSOCIATIVE CLASSIFIER BASED ON FUZZY ASSOCIATION RULE. International Journal of Computational Intelligence Systems, Vol.1, No. 3 (August, 2008), $262-2732008$

[4] Fadi Thabtah, A review of associative classification mining, The Knowledge Engineering Review, Volume 22, Issue 1 (March 2007), Pages 37-65, 2007.

[5] Miguel Delgado, Nicolas Marin, Daniel Sanchez, and maria-Amparo Vila, Fuzzy Association Rules: General Models and Applications, IEEE TRANSACTION ON FUZZY SYSTEM ,VOL 11, NO.2, April 2003.

[6] Khan, M.S. Muyeba, M. Coenen, F A Weighte Utility Framework for Mining Association Rules, Symposium Computer Modeling and Simulation, 2008. EMS '08. Second UKSIM European , page(s): 87-92. 
International Journal of Computer Science, Engineering and Information Technology (IJCSEIT), Vol.2, No.1, February 2012

[7] H. Ishibuchi and T. Yamamoto, "Rule weight specification in fuzzy rule-based classification systems," IEEE Trans. on Fuzzy Systems, vol. 13, no. 4, pp. 428-435, August 2005.

[8] Feng Tao, Fionn Murtagh and Mohsen Farid. Weighted Association Rule Mining using Weighted Support and Significance Framework Proceedings of the ninth ACM SIGKDD international conference on Knowledge discovery and data mining 2003, Pages:661-666 Year of Publication: 2003

[9] Lu, J-J.: Mining Boolean and General Fuzzy Weighted Association Rules in Databases, Systems Engineering-Theory \& Practice, 2, 28--32 (2002)

[10] W. Li, J. Han, and J. Pei. CMAR: Accurate and efficient classification based on multiple classassociation rules. In ICDM'01, pp. 369(376, San Jose, CA, Nov.2001).

[11] Gyenesei, A.: Mining Weighted Association Rules for Fuzzy Quantitative Items, Proceedings of PKDD Conference pp. 416--423 (2000).

[12] Wai-Ho Au Keith C.C. Chan, FARM: A Data Mining System for Discovering Fuzzy Association Rules Proc. of the 8th IEEE Int'l Conf. on Fuzzy Systems, Seoul, Korea 1999.

[13] B. Liu, W. Hsu, and Y. Ma. Integrating Classification and Association Rule Mining. In KDD'98, New York, NY, Aug.1998.

\section{Authors}

Mrs. Sunita Soni is a Sr. Associate Professor in Department of Computer Applications at Bhilai Institute of Technology, Durg (C.G.), India. She is a postgraduate from Pt. Ravi Shankar Shukla University, India. She is a Life fellow member of Indian Society for Technical Education. She has total teaching experience of 12 years He has a total of 16 Research papers published in National / International Journals / Conferences into her credit. Presently She is pursuing PhD from Pt. Ravi Shankar Shukla University, Raipur under the guidance of Dr. O.P.Vyas, IIIT, Allahabad.

Dr.O.P.Vyas is currently working as Professor and Incharge Officer (Doctoral Research Section) in Indian Institute of Information Technology-Allahabad (Govt. of India's Center of Excellence in I.T.). Dr.Vyas has done M.Tech.(Computer Science) from IIT Kharagpur and has done Ph.D. work in joint collaboration with Technical University of Kaiserslautern (Germany) and I.I.T.Kharagpur. With more than 25 years of academic experience Dr.Vyas has guided Four Scholars for the successful award of $\mathrm{Ph} . \mathrm{D}$. degree and has more than 80 research publications with two books to his credit. His current research interests are Linked Data Mining and Service Oriented Architectures.
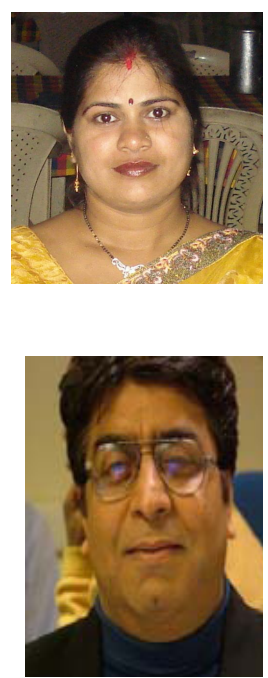